University of Shizuoka

US-95-03

AMU-95-04

Revised Version

October 1995

\title{
Top-Quark-Mass Enhancement in a Seesaw-Type Quark Mass Matrix
}

\author{
Yoshio Koide' \\ Department of Physics, University of Shizuoka \\ 395 Yada, Shizuoka 422, Japan \\ and \\ Hideo Fusaokaf \\ Department of Physics, Aichi Medical University \\ Nagakute, Aichi 480-11, Japan
}

\begin{abstract}
We investigate the implications of a seesaw type mass matrix, i.e., $M_{f} \simeq m_{L} M_{F}^{-1} m_{R}$, for quarks and leptons $f$ under the assumption that the matrices $m_{L}$ and $m_{R}$ are common to all flavors (up-/downand quark-/lepton- sectors) and the matrices $M_{F}$ characterizing the heavy fermion sectors have the form [(unit matrix) $+b_{f}$ (a democratic matrix)] where $b_{f}$ is a flavor parameter. We find that by adjusting the complex parameter $b_{f}$, the model can provide that $m_{t} \gg m_{b}$ while at the same time keeping $m_{u} \sim m_{d}$ without assuming any parameter with hierarchically different values between $M_{U}$ and $M_{D}$. The model with three adjustable parameters under the "maximal" top quark mass enhancement can give reasonable values of five quark mass ratios and four KM matrix parameters.
\end{abstract}

* hep-ph/9505201: To be published in Z. Phys. C.

$\dagger$ E-mail: koide@u-shizuoka-ken.ac.jp

‡E-mail: fusaoka@amugw.aichi-med-u.ac.jp 


\section{Introduction}

One of the most mysterious facts in the quark mass spectrum is why top quark mass $m_{t}$ is so much larger than the bottom quark mass $m_{b}$, while $u$ quark mass $m_{u}$ is of the order of $d$ quark mass $m_{d}$. In the usual discussion of fermion masses, this drastic generation dependence of the mass splitting between members of each isomultiplet of quarks is attributed to an arbitrary hierarchy among the input parameters which is not completely satisfactory. It is therefore important to seek alternative ways to understand this feature. In this paper we argue that within the see-saw[1] type mass formula for quark masses discussed in the context of gauge models [2], a very simple explanation of this feature is obtained by imposing a specific universality ansatz for various flavor matrices. We then find that a slight generalization of this ansatz provides an extremely good fit to all the quark mass ratios and mixings.

Our starting point is the following specific see-saw type ansatz proposed by one of the authors [3] for quark and lepton mass matrices:

$$
M_{f}=M_{e}^{1 / 2} O_{f} M_{e}^{1 / 2}
$$

where $M_{e}^{1 / 2}=\operatorname{diag}\left(\sqrt{m_{e}}, \sqrt{m_{\mu}}, \sqrt{m_{\tau}}\right)$. Here, for the up-quark mass matrix $M_{u}$, the matrix $O_{f}(f=u)$ is given by

$$
O_{f}=\mathbf{1}+3 a_{f} X
$$

where $\mathbf{1}$ is a unit matrix and $X$ is a democratic-type matrix [4]

$$
X=\frac{1}{3}\left(\begin{array}{lll}
1 & 1 & 1 \\
1 & 1 & 1 \\
1 & 1 & 1
\end{array}\right)
$$

which satisfies the relation $X^{2}=X$. The up-quark mass matrix can then successfully give a quark mass ratio $[3,5]$

$$
\frac{m_{u}}{m_{c}} \simeq \frac{3 m_{e}}{4 m_{\mu}}
$$

for a large value of the parameter $a_{u}$. The value of $a_{u}$ is adjusted from the mass ratio $m_{c} / m_{t}$. 
Stimulated by the phenomenological success of the mass matrix form (1.1) - (1.3), the authors [6] have applied the mass matrix form to down-quark mass matrix, by considering that the parameter $a_{d}$ is complex. They have found that the value of a complex parameter $a_{d}$ which fits the mass ratios $m_{d} / m_{s}$ and $m_{s} / m_{b}$ gives reasonable values of not only Kobayashi-Maskawa (KM) [7] matrix elements $V_{i j}(i, j$ denote family indices) but also up-to-down-quark mass ratios $m_{u} / m_{d}, m_{c} / m_{s}$ and $m_{t} / m_{b}$.

Suggested from the form (1.1), it may be expected that such phenomenological success will also be obtained in the context of a seesaw-type mass matrix

$$
M_{f} \simeq m_{L} M_{F}^{-1} m_{R}
$$

with $m_{L} \propto m_{R} \propto M_{e}^{1 / 2}$ and $M_{F} \propto O_{f}^{-1}$. Here, the expression (1.5) is derived from the $6 \times 6$ mass matrix for fermions $(f, F)$

$$
\left(\bar{f}_{L} \bar{F}_{L}\right)\left(\begin{array}{cc}
0 & m_{L} \\
m_{R} & M_{F}
\end{array}\right)\left(\begin{array}{l}
f_{R} \\
F_{R}
\end{array}\right)+\text { h.c. },
$$

for the case of $O\left(M_{F}\right) \gg O\left(m_{R}\right), O\left(m_{L}\right)$, where $f=\left(f_{1}, f_{2}, f_{3}\right)$ are three family quarks and leptons, and $F=\left(F_{1}, F_{2}, F_{3}\right)$ are vector-like heavy fermions corresponding to $f$.

The re-interpretation of the model (1.1) based on the seesaw model (1.5) seems to be plausible because of the following reasons. The inverse of the matrix $O_{f}$ with a simple form [(unit matrix $)+($ democratic-type matrix $\left.)\right]$ has also a simple form $[$ (unit matrix $)+($ democratic-type matrix $)]$, i.e.,

$$
O_{F} \equiv O_{f}^{-1}=\mathbf{1}+3 b_{f} X
$$

where the complex coefficients $a_{f}$ and $b_{f}$ are related by

$$
a_{f}=-b_{f} /\left(1+3 b_{f}\right) .
$$

In the mass matrix model (1.1), we need hierarchically different values [6] of the parameters $a_{f}$, i.e., $a_{u}=28.65$ and $\left|a_{d}\right|=0.4682$, in order to provide reasonable quark masses and KM mixings, while, as seen from (1.8), the values $\left|a_{u}\right| \gg 1$ and $a_{d} \simeq-1 / 2$ correspond to $b_{u} \simeq-1 / 3$ and $b_{d} \simeq-1$ in the inverse matrix (1.7), respectively. In the present paper, we are interested in such a model that $M_{u}$ and 
$M_{d}$ are "almost" symmetric, i.e., they have almost the same structure and they take parameter values which are not so hierarchically different between $M_{u}$ and $M_{d}$. The parameter ratio $\left|a_{u} / a_{d}\right| \simeq 60$ in the model (1.1) can be reduced to the ratio $\left|b_{d} / b_{u}\right| \simeq 3$ in (1.7).

However, when we consider a model (1.6) (not (1.5)) with $M_{F} \propto O_{F}$, one problem arises: Recently the CDF collaboration [8] has reported $m_{t}=174 \pm 10_{-12}^{+13}$ $\mathrm{GeV}$ as top quark mass from $\bar{p} p$ collision data at $\sqrt{s}=1.8 \mathrm{TeV}$. On the other hand, the universal mass matrix $m_{L}$ which breaks the $\mathrm{SU}(2)_{L}$ gauge symmetry should be of the order $\Lambda_{W}=\left(\sqrt{2} G_{F}\right)^{-1 / 2} / \sqrt{2}=174 \mathrm{GeV} \sim m_{t}$, or less. Then, the approximate expression (1.4) for up-quarks is not valid any longer, because if (1.5) is valid, $O\left(m_{L}\right) \sim m_{t}$ means $M_{U}^{-1} m_{R} \sim O(1)$, so that it does not satisfy the condition $O\left(M_{F}\right) \gg O\left(m_{R}\right)$ for the validity of the seesaw expression (1.5). This is also understood from the fact that the limit $\left|a_{u}\right| \rightarrow \infty$ means the limit $b_{u} \rightarrow-1 / 3$ and the determinant of $M_{U}$ becomes zero in the limit, so that the expansion of $M_{f}$ in $M_{F}^{-1}$ can not be a good approximation.

In this paper, we do not use the approximate relation (1.5), but calculate directly the $6 \times 6$ mass matrix (1.6). In Sect. 2, we will give the outline of our mass matrix model. In Sect. 3, we will give an expression of $M_{f}$ which is valid in the limit of $b_{f} \rightarrow-1 / 3$, i.e., $\operatorname{det} M_{F}=0$, instead of the well-known seesaw expression (1.5), and discuss the up-quark mass ratios which are expressed in terms of lepton mass ratios and our adjustable parameters (see the next section). In Sec. 4, we discuss the fermion mass spectra by numerically evaluating the $6 \times 6$ mass matrix. In Sect. 5 , KM matrix parameters are discussed numerically. In the present model, under some basic assumptions (see Sects. 2 and 5), the parameter fitting for quark mass ratios and KM matrix parameters $(5+4=9$ observables) is done by three adjustable parameters $k / K, b_{d}$ and $\beta_{d}$ (see the next section for the definitions). We will find that the value of $m_{t}$ takes the largest enhancement at $b_{u}=-1 / 3$, while the relations $m_{u} \sim m_{d}$ and (1.4) are kept. We can obtain reasonable values of quark mass ratios (not only $m_{u} / m_{c}, m_{c} / m_{t}, m_{d} / m_{s}$ and $m_{s} / m_{b}$, but also $m_{u} / m_{d}, m_{c} / m_{s}$ and $\left.m_{t} / m_{b}\right)$ and the KM matrix parameters, by taking $b_{u}=-1 / 3$ and $b_{d} \simeq-1$.

\section{Outline of the model}

In addition to the conventional quarks and leptons $f_{i}$, where $f$ is the flavor index $(f=u, d, \nu$ and $e$ denote up-quarks, down-quarks, neutrinos and charged leptons), and $i$ is the family index $(i=1,2,3)$, We consider vector-like fermions $F_{i}$ 
correspondingly to $f_{i}$. These fermions belong to $f_{L}=(2,1), f_{R}=(1,2), F_{L}=(1,1)$ and $F_{R}=(1,1)$ of $\mathrm{SU}(2)_{L} \times \mathrm{SU}(2)_{R}$. A "would-be" seesaw mass matrix for the fermions $(f, F)$ is given by (1.6). Gauge models which realize the mass matrix form (1.6) have been proposed by many authors [2]. Although the interest of most authors is how to embed the model (1.6) into a unification model in the framework of gauge theory, our interest is how to give realistic quark mass spectra and family mixing from the phenomenological point of view.

Suggested by the phenomenological success of the model (1.1), we assume the following mass matrix [9]

$$
M=\left(\begin{array}{cc}
0 & m_{L} \\
m_{R} & M_{F}
\end{array}\right)=m_{0}\left(\begin{array}{cc}
0 & Z \\
k Z & K O_{F}
\end{array}\right)
$$

where the matrices $m_{L}$ and $m_{R}$ (i.e., $m_{0}, h$ and the matrix $Z$ ) are common to all of $f=u, d, \nu, e$, and only $M_{F}$ depends on flavors $f$ through the complex parameter $b_{f}$. Hereafter, we denote the complex parameter $b_{f}$ in (1.7) as $b_{f} e^{i \beta}\left(b_{f}\right.$ is real and $\left.\left|\beta_{f}\right| \leq \pi / 2\right)$ in (2.2) below. The vector-like fermions $F$ acquire large masses $M_{F}$ at an energy scale $\mu=m_{0} K$. We consider that the energy scale $m_{0} K$ is not as large as the ground unification scale, but an intermediate energy scale. At the present stage, the origin of the democratic form

$$
O_{F}=1+3 b_{f} e^{i \beta_{f}} X=\left(\begin{array}{ccc}
1 & 0 & 0 \\
0 & 1 & 0 \\
0 & 0 & 1
\end{array}\right)+b_{f} e^{i \beta_{f}}\left(\begin{array}{lll}
1 & 1 & 1 \\
1 & 1 & 1 \\
1 & 1 & 1
\end{array}\right)
$$

is an open question. We may attribute the origin of the democratic term $X$ to a permutation symmetry $\mathrm{S}_{3}$ [10], a BCS-like mechanism [11], a composite model based on the analogy of hadronic $\pi^{0}-\eta-\eta^{\prime}$ mixing [12], and so on. In the present phenomenological analysis, we do not discuss its origin moreover.

The present model is left-right symmetric except for $k \neq 1$. At an energy scale $\mu=m_{0} k\left(\mu=m_{0}\right)$ at which $\mathrm{SU}(2)_{R}\left(\mathrm{SU}(2)_{L}\right)$ is broken, the mass term $\bar{F}_{L} m_{R} f_{R}\left(\bar{f}_{L} m_{L} F_{R}\right)$ appears, so that we consider $k \sim m\left(W_{R}\right) / m\left(W_{L}\right)$. The relation $m_{L}=m_{R} / k=m_{0} Z$ is merely a phenomenological working hypothesis. The matrix $Z$ takes a diagonal form

$$
Z=\operatorname{diag}\left(z_{1}, z_{2}, z_{3}\right)
$$


with the normalization condition $z_{1}^{2}+z_{2}^{2}+z_{3}^{2}=1$. (In other words, in the family basis in which $Z$ is diagonal, we have assumed that the matrix $O_{F}$ is given by (2.2)). For the charged leptons, since $m_{\tau} \ll m_{0} \sim m_{W}$, it is clear that the seesaw expression $M_{e}=m_{0}(k / K) Z O_{F}^{-1} Z$ is well satisfied, so that we can fix the parameter $z_{i}$ as

$$
\frac{z_{1}}{\sqrt{m_{e}}}=\frac{z_{2}}{\sqrt{m_{\mu}}}=\frac{z_{3}}{\sqrt{m_{\tau}}}=\frac{1}{\sqrt{m_{e}+m_{\mu}+m_{\tau}}} .
$$

Here, we have assumed $b_{e}=0$ according to the phenomenological success [3] of the model (1.1). In the present paper, we do not discuss why $z_{i}$ are given by the relation (2.4), because the purpose of the present paper is to study quark mass ratios and KM matrix parameters phenomenologically, so that charged lepton masses are regarded as inputs in the numerical estimates. Since the evolution effects of fermion mass ratios (not the absolute values) from $\mu=m_{0} K$ to $\mu=m_{0}$ are, at most, several percent, for simplicity, we use the values of $z_{i}$ which are fixed by using the formula (2.4) with the observed charged lepton masses [13].

For the case of $K \gg k \gg 1$, the quark mass ratios and the KM matrix parameters (nine observables) are described by five real parameters $k / K$ (not $k$ and $K$ separately), $b_{u}, \beta_{u}, b_{d}$ and $\beta_{d}$. As we will discuss in Sections 3 and 4 , the maximal top-quark-mass enhancement occurs at $b_{u}=-1 / 3$ and $\beta_{u}=0$. We will put an ansatz of "maximal top-quark-mass enhancement", so that we will fix the parameters $b_{u}$ and $\beta_{u}$ to $b_{u}=-1 / 3$ and $\beta_{u}=0$. The numerical fitting for the nine observables is then tried by adjusting only three parameters $k / K, b_{d}$ and $\beta_{d}$. However, as will be discussed in Sect. 5, a straightforward application of the mass-matrix model (2.1) cannot lead to reasonable predictions of the KM matrix parameters. We will therefore introduce a sign factor by replacing $m_{L}=m_{0} Z$ in (2.1) by $m_{L}^{f}=m_{0} P_{f} Z$, where $P_{u}=\operatorname{diag}(1,1,1)$, while $P_{d}=\operatorname{diag}(1,1,-1)$. The adjustable parameters are still three, i.e., $k / K, b_{d}$ and $\beta_{d}$. The phase matrices $P_{f}$ do not affect the discussion of the mass spectrum. For a time being in Sects. 3 and 4, we will neglect the phase matrices $P_{f}$.

\section{Expression of $M_{f}$ in the case of $b_{f} \simeq-1 / 3$}

One of the purposes in the present paper is to obtain a reliable expression of $M_{f}$ in the case of $b_{f} \simeq-1 / 3$, because the case leads to $\operatorname{det} M_{F} \simeq 0$, so that the seesaw expression (1.5) which is obtained by expanding it in $M_{F}^{-1}$ is not valid any 
longer.

As shown in Appendix, in general, the transformation of the $6 \times 6$ mass matrix $M$ into

$$
U_{L} M U_{R}^{\dagger} \equiv U_{L}\left(\begin{array}{cc}
M_{11} & M_{12} \\
M_{21} & M_{22}
\end{array}\right) U_{R}^{\dagger}=M^{\prime} \equiv\left(\begin{array}{cc}
M_{11}^{\prime} & 0 \\
0 & M_{22}^{\prime}
\end{array}\right)
$$

is done by the following two $6 \times 6$ unitary matrices,

$$
U_{L}=\left(\begin{array}{cc}
\left(1+\rho_{L} \rho_{L}^{\dagger}\right)^{-1 / 2} & \left(1+\rho_{L} \rho_{L}^{\dagger}\right)^{-1 / 2} \rho_{L} \\
-\left(1+\rho_{L}^{\dagger} \rho_{L}\right)^{-1 / 2} \rho_{L}^{\dagger} & \left(1+\rho_{L}^{\dagger} \rho_{L}\right)^{-1 / 2}
\end{array}\right)
$$

and $U_{R}$ with $L \leftrightarrow R$ in (3.2). The so-called seesaw expression $M_{11}^{\prime} \equiv M_{f} \simeq$ $m_{L} M_{F}^{-1} m_{R}$ is obtained by expanding $M_{11}^{\prime}$ in $M_{F}^{-1}$. Since our mass matrix (2.1) is not Hermitian, for evaluating the KM matrix (family mixing of left-handed fermions), it is useful to define the $3 \times 3$ Hermitian matrix $H_{f}$ :

$$
H_{f} \equiv M_{11}^{\prime} M_{11}^{\prime \dagger}=\left(1+\rho_{L} \rho_{L}^{\dagger}\right)^{-1 / 2} \widetilde{H}_{f}\left(1+\rho_{L} \rho_{L}^{\dagger}\right)^{+1 / 2}
$$

As seen in (A.22), (A.24) and (A.27), the matrix $\widetilde{H}_{f}$ is given by

$$
\widetilde{H}_{f} \equiv \rho_{L} m_{R} \rho_{R} m_{L}^{\dagger}=\left(m_{L}+\rho_{L} M_{F}\right) m_{L}^{\dagger},
$$

and it satisfies the following equation:

$\widetilde{H}_{f}^{2} m_{L}^{\dagger-1}-\widetilde{H}_{f} m_{L}^{\dagger-1}\left(M_{F}^{\dagger} M_{F}+m_{L}^{\dagger} m_{L}+M_{F}^{-1} m_{R} m_{R}^{\dagger} M_{F}\right)+m_{L} M_{F}^{-1} m_{R} m_{R}^{\dagger} M_{F}=0$.

Our interest is in the expression of $\widetilde{H}_{f}$ in the case of $\operatorname{det} M_{F} \simeq 0$. However, since it is hard to obtain the general formulation in such the case, we confine ourselves to investigating the special form (2.1) with (2.2).

For the investigation of the case of $b_{u} \simeq-1 / 3$, it is convenient to define the parameter

$$
3 \varepsilon \equiv \Delta b=b+\frac{1}{3}
$$

Then, the matrix $O_{F}$ is represented by

$$
O_{F}=Y+\varepsilon X,
$$


where

$$
Y=1-X,
$$

and the matrices $X$ and $Y$ satisfy the relations $X^{2}=X, Y^{2}=Y$, and $X Y=$ $Y X=0$ from the definitions (1.3) and (3.8), so that the inverse of $O_{F},(3.7)$, is given by

$$
O_{F}^{-1}=Y+X / \varepsilon .
$$

For the case of $(k / K)^{2} \ll \varepsilon^{2} \ll 1$, from the equation (3.5), we obtain

$$
\widetilde{H}_{f} \simeq m_{0}^{2}\left(\frac{k}{K}\right)^{2} Z\left(Y+\frac{1}{\varepsilon} X\right) Z^{2}\left(Y+\frac{1}{\varepsilon} X\right) Z
$$

which corresponds to the well-known seesaw expression $M_{f} \simeq m_{0}(k / K) Z O_{F}^{-1} Z$.

For a general case, we assume an approximate form

$$
\widetilde{H}_{u} \simeq m_{0}^{2} Z\left(\frac{k}{K} Y+x X\right) Z^{2}\left(\frac{k}{K} Y+x X\right) Z,
$$

from an analogy to the form (3.10). By substituting (3.11) into (3.5), we find

$$
x \simeq\left[\frac{\varepsilon}{2 k / K}+\sqrt{\frac{1}{3}+\left(\frac{\varepsilon}{2 k / K}\right)^{2}}\right]^{-1} .
$$

For $\varepsilon^{2} \gg(k / K)^{2},(3.12)$ reproduces (3.10). For $\varepsilon^{2} \ll(k / K)^{2}$, we obtain

$$
\widetilde{H}_{u} \simeq 3 m_{0}^{2} Z\left(X+\frac{1}{\sqrt{3}} \frac{k}{K} Y\right) Z^{2}\left(X+\frac{1}{\sqrt{3}} \frac{k}{K} Y\right) Z .
$$

This expression (3.13) is the expression which should be used in the case of $\operatorname{det} M_{F} \simeq$ 0 as a substitute for the well-known seesaw expression (3.10).

The mass eigenvalues are calculated from $\operatorname{Tr} H_{u}=\operatorname{Tr} \widetilde{H}_{u},\left(\left(\operatorname{Tr} H_{u}\right)^{2}-\operatorname{Tr} H_{u}^{2}\right) / 2=$ $\left(\left(\operatorname{Tr} \widetilde{H}_{u}\right)^{2}-\operatorname{Tr} \widetilde{H}_{u}^{2}\right) / 2$ and $\operatorname{det} H_{u}=\operatorname{det} \widetilde{H}_{u}$. We obtain up-quark masses

$$
m_{u} \simeq \frac{3}{2} z_{1}^{2} \frac{k}{K} m_{0}, \quad m_{c} \simeq 2 z_{2}^{2} z_{3}^{2} \frac{k}{K} m_{0}, \quad m_{t} \simeq \frac{1}{\sqrt{3}} \frac{1}{\sqrt{1+27(\Delta b)^{2}(K / k)^{2}}} m_{0},
$$


from (3.11) and (3.12), where $\varepsilon=\Delta b / 3$ (3.6). We find that the relation (1.4) is also valid in the case $(\Delta b)^{2} \ll(k / K)^{2} \ll 1$, even in the limit of $b_{u}=-1 / 3$.

\section{Numerical study of quark mass ratios}

Numerical evaluation of the eigenvalues of the $6 \times 6$ mass matrix (2.1) can easily be done with the help of a computer. Numerical study is helpful for checking analytical calculations based on the formalism of the previous section. In Fig. 1, in order to give an overview of the mass spectrum in our mass matrix model, we illustrate the light fermion mass spectrum $m_{i}^{f}(i=1,2,3)$ versus the parameter $b_{f} e^{i \beta_{f}}$. Here, we have taken $k=10$ and $K / k=50$ as a trial. (The choices of $k$ and $K / k$ are discussed later.) In order to fix the values of the parameters $z_{i}$ at $b_{e}=0$, we have used the observed charged leptons masses [13] as inputs.

The spectrum for the case of $\beta_{f}=0$ (solid lines) shows the following characteristics:

(1) The third fermion mass is sharply enhanced at $b_{f}=-1 / 3$.

(2) Level crossing (mass degeneration) occurs at $b_{f}=-1 / 2$ and $b_{f}=-1$.

These characteristics become mild when $\beta_{f}$ takes a sizable value (dashed lines).

For comparison, we list the observed running quark mass values (in unit of $\mathrm{GeV})[14]$ at $\mu=\Lambda_{W} \equiv\left(\sqrt{2} G_{F}\right)^{-1 / 2}=174 \mathrm{GeV}$ :

$$
\begin{array}{lll}
m_{u}=0.00230 \pm 0.00045, & m_{c}=0.612_{-0.023}^{+0.010}, & m_{t}=166_{-26}^{+21}, \\
m_{d}=0.00406 \pm 0.00045, & m_{s}=0.082 \pm 0.014, & m_{b}=2.874_{-0.023}^{+0.012}
\end{array} .
$$

In the previous section, we have showed that the up-quark mass ratio $m_{u} / m_{c}$ is given by (1.4) in the limit $\varepsilon \ll(k / K)^{2} \ll 1$, see (3.14). The relation can be checked by a numerical study. We find that the ratio $m_{c} / m_{u}$ at a fixed $K / k$ is insensitive to the choice of $k$, for $k \geq 10$. Also, the ratio is insensitive to the parameters $K / k$ and $\Delta b_{u}$ for large $K / k$; for example, $m_{c} / m_{u}=260.8,260.8,259.2$, and 259.2, for $\left(K, k, \Delta b_{u}\right)=\left(10^{3}, 10,0\right),\left(10^{5}, 10,0\right),\left(10^{3}, 10,0.003\right)$ and $\left(10^{5}, 10,0.003\right)$, respectively, while $\left(m_{c} / m_{u}\right)_{\exp }=266_{-49}^{+70}$. Thus, we conclude that the relation (1.4) is valid almost independently of the values of $k$ and $K / k$ for the case of $K \gg k \gg 1$.

Next, we study the up-quark mass ratio $m_{t} / m_{c}$. We find that the ratio is also insensitive to the value of $k$ for $k \geq 10$. Therefore, we illustrate the behavior of $m_{t} / m_{c}$ versus $K / k$ for the case of $k=10$ in Fig. 2. It is noticeable that, for $\Delta b_{u} \simeq$ +0.00388 and $\Delta b_{u} \simeq-0.00362$, the ratio $m_{t} / m_{c}$ comes near the experimental value 
$\left(m_{t} / m_{c}\right)_{e x p} \simeq 271$ as $K / k \rightarrow \infty$. For the case $\left|\Delta b_{u}\right| \geq 0.005$, we cannot fit the ratio $m_{c} / m_{t}$ suitably, so that the case is ruled out. For $\Delta b_{u}=0$, the ratio $m_{t} / m_{c}$ increases linearly in $K / k$. In order to fit the prediction to the experimental value of $\left(m_{t} / m_{c}\right)_{\text {exp }}=271 \pm 46$, we need $K / k=50 \pm 8$ for the case $\Delta b_{u}=0$ whereas we find $K / k=\left(2.0_{-1.3}^{+\infty}\right) \times 10^{2}$ for the cases $\Delta b_{u}=+0.00388$ and $\Delta b_{u}=-0.00362$.

Although a scenario with $\Delta b_{u} \simeq \pm 0.004$ and $K / k>2 \times 10^{2}$ seems to be attractive because the ratio $m_{t} / m_{c}$ can be fitted insensitive to $K / k$, we do not adopt this scenario because of the following consideration of the absolute value of $m_{t}$. In Fig. 3, we show the behavior of $m_{t} / m_{0}$ versus $K / k$. Since the ratio is again insensitive to the value of $k$ for $k \geq 10$, we illustrate the case of $k=10$. In the limit of $b_{u}=-1 / 3$, the value $m_{t} / m_{0}$ is almost constant, i.e., $m_{t} / m_{0} \simeq 1 / \sqrt{3}$ (a) as we have shown in (3.14). On the other hand, as seen in Fig. 3, the case (c) $\Delta b_{u} \simeq \pm 0.004$ gives $m_{t} / m_{0}<0.161$ for $K / k>2 \times 10^{2}$. If we consider that the mass matrix $m_{L}$ originates from the couplings to an $\mathrm{SU}(2)_{L}$ doublet Higgs boson $\phi_{L}$ with the vacuum expectation value (VEV) $\left\langle\phi_{L}^{0}\right\rangle_{0}=v_{0}=\Lambda_{W}=174 \mathrm{GeV}$, the Yukawa coupling constants $y_{L i}$ with fermions $\bar{f}_{L i} F_{R i}$ are given by $y_{L i}=z_{i} m_{0} / v_{0}$. Therefore, a small value of $m_{t} / m_{0}$ means a large value of $y_{L 3}=z_{3}\left(m_{0} / m_{t}\right)\left(m_{t} / v_{0}\right)$. The value $m_{t} / m_{0}=0.161$ corresponds to $y_{L 3}=6.03\left(m_{t} / v_{0}\right)$. Such a large value may be unfavorable from the point of view of the perturbative electroweak theory. Hereafter, we adopt the ansatz of the "maximal top-quark-mass enhancement", i.e., $b_{u}=-1 / 3$ (solid lines in Figs. 2 and 3), and we fix the parameter $K / k$ to $K / k=50$ from the observed ratio of $m_{t} / m_{c}$.

On the other hand, the down-quark masses are given by adjusting two parameters $b_{d}$ and $\beta_{d}$. As seen in Fig. 1, the case of $b_{d} \simeq-1$ is favorable because it can give reasonable predictions not only for $m_{b} / m_{s}$ and $m_{s} / m_{d}$, but also for $m_{d} / m_{u}$. The ratios $m_{s} / m_{d}$ and $m_{b} / m_{s}$ versus $b_{d}$ and $\beta_{d}$ are illustrated in Fig. 4 for the case of $K / k=50$ and $k=10$. As far as we see in Fig. 4 , the cases $b_{d}=-1.1 \sim-1$ with $\beta_{d}=-20^{\circ} \sim-16^{\circ}$ are favorable. Considering the present experimental uncertainty of quark mass values, hereafter, we simply adopt the integral solution $b_{d}=-1$ for further numerical estimates.

For the case of $b_{d} \simeq-1$ and $1 \gg \beta_{d}^{2} \neq 0$, down-quark masses are given by

$$
m_{d} \simeq z_{1}^{2} \frac{1}{\beta_{d}} \frac{k}{K} m_{0}, \quad m_{s} \simeq z_{2}^{2} z_{3}^{2} \beta_{d} \frac{k}{K} m_{0}, \quad m_{b} \simeq \frac{1}{2} \frac{k}{K} m_{0} .
$$


In the present model, the up-to-down quark mass ratio $m_{u} / m_{d}$ is given by

$$
\frac{m_{u}}{m_{d}} \simeq 3 \frac{m_{s}}{m_{c}} \simeq \frac{3}{2} \beta_{d},
$$

so that the ratios $m_{u} / m_{d}$ and $m_{s} / m_{c}$ can be fitted independently of $m_{t} / m_{c}$ (i.e., $K / k)$ by adjusting the parameter $\beta_{d}$.

When we take $b_{d}=-1.0$ and $\beta_{d}=-18^{\circ}$ (and $k=10$ and $K / k=50$ ), we can obtain reasonable quark mass values:

$$
\begin{array}{lll}
m_{u}\left(\Lambda_{W}\right)=0.00234 \mathrm{GeV}, & m_{c}\left(\Lambda_{W}\right)=0.610 \mathrm{GeV}, & m_{t}\left(\Lambda_{W}\right)=166 \mathrm{GeV}, \\
m_{d}\left(\Lambda_{W}\right)=0.00475 \mathrm{GeV}, & m_{s}\left(\Lambda_{W}\right)=0.0923 \mathrm{GeV}, & m_{b}\left(\Lambda_{W}\right)=3.01 \mathrm{GeV}
\end{array}
$$

where we have taken $m_{0}\left(\Lambda_{W}\right)=288 \mathrm{GeV}$ to have $m_{t}\left(\Lambda_{W}\right)=166 \mathrm{GeV}$.

So far, except for (4.4), we have discussed only quark mass ratios and not the absolute values, because the ratios are comparatively insensitive to the evolution from $\mu=m_{0} K$ to $\mu=m_{0}$. The common value $m_{0}\left(\Lambda_{W}\right)=288 \mathrm{GeV}$ does not give the absolute magnitudes of the charged lepton masses, $(k / K) m_{0}=m_{\tau}+m_{\mu}+m_{e}$. We find

$$
\left.\frac{\left(m_{0} k / K\right)_{q}}{\left(m_{0} k / K\right)_{\ell}}\right|_{\mu=\Lambda_{W}}=3.05,
$$

where $\left(m_{0} k / K\right)_{q(\ell)}$ denotes the value of $m_{0} k / K$ in the quark (lepton) sector. It is not likely that the factor 3.1 comes only from the evolution from $\mu=m_{0} K$ to the present scale $\mu=\Lambda_{W}$. Since we consider the case where the parameters $m_{0}$ and $k$ (i.e., $m_{L}$ and $m_{R}$ ) are universal for all flavors $f=u, d, \nu, e$, the discrepancy (4.5) should come from the difference in $K$ between the quark- and lepton-sectors, i.e., $K_{q} \neq K_{\ell}$. Although it is possible that the coupling constants of the colored heavy fermions with Higgs bosons which generate the democratic-type matrix (2.2) are smaller than that of the colorless heavy fermions by a factor $1 / 3$, i.e., $K_{\ell} / K_{q}=3$, we do not discuss the origin of $K_{\ell} / K_{q}=3$ in the present paper. In the present model, we practically consider that $m_{L}$ and $m_{R}$ are universal for quarks and leptons, while $M_{F}$ are not so, and $K_{u}=K_{d} \equiv K_{q} \neq K_{\nu}=K_{e} \equiv K_{\ell}$. Hereafter, we denote $K_{q}$ simply as $K$. 
Similarly, with the same parameter values as in (4.4), the heavy quark masses are given as follows:

$$
\begin{array}{lll}
m_{4}^{u}\left(\Lambda_{W}\right)=1.66 \mathrm{TeV}, & m_{5}^{u}\left(\Lambda_{W}\right)=144 \mathrm{TeV}, & m_{6}^{u}\left(\Lambda_{W}\right)=144 \mathrm{TeV} \\
m_{4}^{d}\left(\Lambda_{W}\right)=144 \mathrm{TeV}, & m_{5}^{d}\left(\Lambda_{W}\right)=144 \mathrm{TeV}, & m_{6}^{d}\left(\Lambda_{W}\right)=298 \mathrm{TeV}
\end{array}
$$

These numerical results are also obtained from the approximate relations for $b_{u}=$ $-1 / 3$ and $b_{d}=-1$ :

$$
\begin{gathered}
m_{4}^{u} \simeq(k / \sqrt{3}) m_{0}, \quad m_{5}^{u} \simeq m_{6}^{u} \simeq K m_{0}, \\
m_{4}^{d} \simeq m_{5}^{d} \simeq K m_{0}, \quad m_{6}^{d} \simeq 2 \sqrt{1+3 \beta_{d}^{2} / 4} K m_{0} .
\end{gathered}
$$

Note that the fourth up-quark $u_{4}$ becomes considerably lighter than the other heavy quarks, at the cost of the enhancingthe top-quark mass. The absolute magnitudes the heavy quark masses in (4.6) should not be taken solidly, because they depend on both $k$ and $K$. We have chosen $K / k=50$ in order to fit $m_{t} / m_{c}$, but the choice $k=10$ was only a trial choice, because the predictions for light fermions (quarks and leptons) are insensitive to the value of $k$. Only constraint on the value $k$ comes from the relation $k \sim m\left(W_{R}^{ \pm}\right) / m\left(W_{L}^{ \pm}\right)$. The present lower bound of the righthanded weak boson mass $m\left(W_{R}\right)$ is given in Ref. [15], so that we cannot choose too small value of $k$. Since $m_{4}^{u}$ is of the order of $k m_{0}$, as seen in (4.7), we can expect to observe the fourth up-quark at the energy scale where the right-handed weak bosons $W_{R}$ are observed.

\section{KM matrix parameters}

In the present model, the parameter fitting for five quark-mass ratios and four KM matrix parameters is done by five parameters, $k / K$ (not $k$ and $K$ ), $b_{u}, \beta_{u}$, $b_{d}$ and $\beta_{d}$. When we adopt the ansatz of "maximal top-quark-mass enhancement", we have fixed the parameters $b_{u}$ and $\beta_{u}$ to $b_{u}=-1 / 3$ and $\beta_{u}=0$, and the remaining adjustable parameters are $k / K, b_{d}$ and $\beta_{d}$. We have pointed out that the relation between up-quark mass ratio $m_{u} / m_{c}$ and $m_{e} / m_{\mu},(1.4)$, is satisfied independently of these parameters for the case $b_{u} \simeq-1 / 3$. The parameter $K / k$ was fixed to $K / k=50$ from the observed up-quark mass ratio $m_{t} / m_{c}$, see Fig. 2 . In the previous section, we have shown that the remaining two parameter $b_{d}$ and $\beta_{d}$ can be fitted to three observed quark mass ratios $m_{d} / m_{s}, m_{s} / m_{b}$ and $m_{u} / m_{d}$ reasonably (see Fig. 4). Then, our final task in the present phenomenological study is to check 
whether these parameter values can also give reasonable predictions for the four KM matrix parameters.

The KM matrix $V$ is given by $V=U_{u} U_{d}^{\dagger}$, where $U_{q}(q=u, d)$ are the unitary matrices to diagonalize the light fermion mass matrices $M_{f} M_{f}^{\dagger}$, where $M_{f} \equiv M_{11}^{\prime}$ $(f=u, d)$ defined by (3.2). Unfortunately, our parameter values $K / k \simeq 50, b_{d} \simeq$ -1 and $\beta_{d} \simeq-18^{\circ}$ give rise to the KM matrix parameters far away from the observed values [13]. Therefore, we must slightly modify our model.

So far, we have assumed that the matrices $m_{L}$ and $m_{R}$ are universal for upand down-sectors. However, in the present section, let us distinguish the matrix $m_{L}$ in the up-quark sector, $m_{L}^{u}=m_{0} Z_{u}$, from that in down-quark sector, $m_{L}^{d}=m_{0} Z_{d}$. We assume that $Z_{u}$ and $Z_{d}$ are given by $Z_{q}=P_{q} Z(q=u, d)$, where $Z$ is given by (2.3) and (2.4), and $P_{q}$ are phase matrices. (It is not essential whether we also assume a similar modification on $m_{R}$ or not, because the KM matrix is related only to the family mixing among the left-handed fields.) Such a modification does not change our predictions on the fermion masses in Sects. 3 and 4, while the KM matrix $V$ is changed into the following expression:

$$
V=U_{u} P U_{d}^{\dagger}
$$

where $U_{q}(q=u, d)$ are unitary matrices to diagonalize the unchanged matrices $M_{f} M_{f}^{\dagger}$ (i.e., in the case of $P_{u}=P_{d}=\mathbf{1}$ ), and $P=P_{u} P_{d}^{\dagger}$. In general, the phase matrix $P$ can have two independent phase parameters such as $P=\operatorname{diag}\left(1, e^{i \delta_{2}}, e^{i \delta_{3}}\right)$. However, since we do not want more adjustable parameters, we examine a simpler ansatz that the phase matrix $P$ is real, i.e., $\delta_{i}=0$ or $\pi$. Thus, we keep three adjustable parameters, $k / K, b_{d}$ and $\beta_{d}$, at the cost of putting the additional ansatz on $P$.

As a result, we find that only for the case

$$
P=\operatorname{diag}(1,1,-1),
$$

we can obtain reasonable values of $\left|V_{u s}\right|,\left|V_{c b}\right|$ and $\left|V_{u b}\right|$. We show $\left|V_{u s}\right|,\left|V_{c b}\right|$ and $\left|V_{u b}\right|$ versus $\beta_{d}$ in Fig. 5 . The same parameter values as in (4.4), $K / k=50, b_{d}=-1$ and $\beta_{d}=-18^{\circ}$, give reasonable predictions

$$
\begin{gathered}
\left|V_{u s}\right|=0.220, \quad\left|V_{c b}\right|=0.0598, \quad\left|V_{u b}\right|=0.00330, \quad\left|V_{t d}\right|=0.0155 \\
J=-3.18 \times 10^{-5}
\end{gathered}
$$


where $J$ is the rephasing invariant [16] $J=\operatorname{Im}\left(V_{c b} V_{u s} V_{c s}^{*} V_{u b}^{*}\right)$. Although the origin of the phase inversion $P=\operatorname{diag}(1,1,-1)$ is not clear and the predicted value of $V_{c b}$ is somewhat large, it is a noticeable feature of the present model that the parameters which were fixed by the observed quark-mass ratios can roughly give reasonable predictions for all the KM matrix parameters.

\section{Conclusions}

In conclusion, we have demonstrated that the seesaw-type mass matrix (2.1) with $M_{F}$ given by (2.2) can give top-quark-mass enhancement without assuming any parameters with hierarchically different values between $M_{U}$ and $M_{D}$, i.e., with $b_{u} \simeq-1 / 3$ and $b_{d} \simeq-1$. The enhancement $m_{t} / m_{b} \gg 1$ comes from the fact that the democratic part $X$ in the inverse matrix $M_{F}^{-1}$ in (1.2), is enhanced as to $b_{f} \rightarrow-1 / 3$ because $\left|a_{f}\right| \rightarrow \infty$ in the limit as seen in (1.8). On the other hand, the result $m_{u} \sim m_{d}$ comes from the feature that the democratic-type mass matrix can give rise to a large mass only to the third family, i.e., the effect of $\left|a_{u}\right| \rightarrow \infty$ contributes mainly to $m_{t}$.

In the present model, the parameter fitting for the five quark mass ratios and the four KM matrix parameters has been done by five parameters $k / K$ (not $k$ and $K$ separately), $b_{u}, \beta_{u}, b_{d}$ and $\beta_{d}$. (The parameters $z_{i}$ were fixed by charged lepton masses.) When we adopt the ansatz of "maximal top-quark-mass enhancement", the parameters $b_{u}$ and $\beta_{u}$ are fixed to $b_{u}=-1 / 3$ and $\beta_{u}=0$, and the remaining adjustable parameters are $k / K, b_{d}$ and $\beta_{d}$. The parameter $K / k$ is then fixed by the observed up-quark-mass ratio $m_{t} / m_{c}$ to be $K / k=50$. The remaining two parameters $b_{d}$ and $\beta_{d}$ are then free parameters by which four quark mass ratios $m_{u} / m_{c}, m_{d} / m_{s}, m_{s} / m_{b}$ and $m_{u} / m_{d}$, and four KM parameters are fitted. As shown in Sects. 4 and 5 , by choosing $b_{d} \simeq-1$ and $\beta_{d} \simeq-18^{\circ}$, we have obtained reasonable fitting for the quark-mass ratios, and also for the KM matrix parameters with the ansatz (5.2).

A few remarks are in order.

In the present model, flavor changing neutral currents (FCNC) can, in principle, appear. However, the FCNC due to the $\mathrm{SU}(2)_{L}\left(\mathrm{SU}(2)_{R}\right)$ doublet Higgs boson exchange through $f-F$ mixing are highly suppressed by a GIM-like mechanism [17]. The FCNC due to the $Z$-boson exchange through $f$ - $F$ mixing are also suppressed because the effective coupling constants are order of $1 / K$ (we can find that those are of the order of $10^{-8}$ in the case of $k=10$ ), so that the FCNC rare decay modes 
are suppressed by $10^{-16}$.

The $C P$ violating phases come only from the heavy fermion mass matrix $M_{F}$, i.e., from the parameter $\beta_{f}$. In the up-quark sector, the parameter $\beta_{u}$ must be $\beta_{u}=0$, because the top quark mass enhancement becomes mild when $\beta_{u} \neq 0$. On the other hand, if $\beta_{d}=0$, we cannot fit down-quark mass ratios $m_{d} / m_{s}$ and $m_{s} / m_{b}$ for any values of $k / K$ and $b_{d}$. We must choose a sizable value of $\beta_{d}$. Thus, in our model, the $C P$ violating phase in quarks comes only from the down-quark sector $M_{D}$.

In the present paper, we have discussed a seesaw mass matrix model with the form of $M_{F}=m_{0} K O_{F}$ given by (2.2). As far as the phenomenological predictions are concerned, we can choose other family-basis, for example, a rather simple form of $O_{F}$

$$
O_{F}=1+3 b_{f} e^{i \beta_{f}} \operatorname{diag}(0,0,1),
$$

instead of the democratic form (2.2). However, in order to obtain reasonable predictions of quark mass ratios and KM matrix parameters, the matrix $Z$ cannot be a diagonal form such as in (2.3), and it must be given by

$$
Z=\frac{1}{6}\left(\begin{array}{ccc}
3\left(z_{2}+z_{1}\right) & -\sqrt{3}\left(z_{2}-z_{1}\right) & -\sqrt{6}\left(z_{2}-z_{1}\right) \\
-\sqrt{3}\left(z_{2}-z_{1}\right) & 4 z_{3}+z_{2}+z_{1} & -\sqrt{2}\left(2 z_{3}-z_{2}-z_{1}\right) \\
-\sqrt{6}\left(z_{2}-z_{1}\right) & -\sqrt{2}\left(2 z_{3}-z_{2}-z_{1}\right) & 2\left(z_{3}+z_{2}+z_{1}\right)
\end{array}\right)
$$

where $z_{i}$ are given by (2.4). Which family basis is reasonable is not essential as far as we discuss only the fermion masses and KM mixing parameters, but it will become important for model-building.

We believe that our phenomenological mass-matrix model is worth serious attention, not only because it has fewer adjustable parameters than conventional models do, but also because it gives $m_{t} \gg m_{b}$ and $m_{u} \sim m_{d}$ simultaneously despite its "almost" up-down symmetric mass matrices (i.e., $b_{u} / b_{d}$ is not so large as $m_{t} / m_{b}$ ). 


\section{Acknowledgments}

The authors would like to express their sincere thanks Professors R. Mohapatra for a careful reading of the original manuscript and providing valuable comments and Professor M. Tanimoto for helpful discussions. The authors are also indebted to Professors K. Matumoto for his encouragement and his stimulating comments. This work was supported by the Grant-in-Aid for Scientific Research, Ministry of Education, Science and Culture, Japan (No.06640407).

\section{Appendix: Diagonalization of $2 n \times 2 n$ matrix}

The transformation of $2 n \times 2 n$ matrix

$$
M=\left(\begin{array}{ll}
M_{11} & M_{12} \\
M_{21} & M_{22}
\end{array}\right)
$$

into

$$
M^{\prime} \equiv\left(\begin{array}{cc}
M_{11}^{\prime} & 0 \\
0 & M_{22}^{\prime}
\end{array}\right)
$$

is done by two $2 n \times 2 n$ unitary matrices,

$$
U_{L}=\left(\begin{array}{cc}
\left(1+\rho_{L} \rho_{L}^{\dagger}\right)^{-1 / 2} & \left(1+\rho_{L} \rho_{L}^{\dagger}\right)^{-1 / 2} \rho_{L} \\
-\left(1+\rho_{L}^{\dagger} \rho_{L}\right)^{-1 / 2} \rho_{L}^{\dagger} & \left(1+\rho_{L}^{\dagger} \rho_{L}\right)^{-1 / 2}
\end{array}\right)
$$

and $U_{R}$ with $L \leftrightarrow R$ in (A.3) as

$$
M^{\prime}=U_{L} M U_{R}^{\dagger}
$$

where $M_{i j}, M_{i j}^{\prime}, \rho_{L}, \rho_{R}$ are $n \times n$ matrices.

The conditions $M_{12}^{\prime}=0$ and $M_{21}^{\prime}=0$ lead to the relations

$$
M_{12}-M_{11} \rho_{R}+\rho_{L} M_{22}-\rho_{L} M_{21} \rho_{R}=0,
$$

and

$$
M_{21}+M_{22} \rho_{R}^{\dagger}-\rho_{L}^{\dagger} M_{11}-\rho_{L}^{\dagger} M_{12} \rho_{R}^{\dagger}=0,
$$

respectively, which lead to

$$
\rho_{R}=\left(M_{11}+\rho_{L} M_{21}\right)^{-1}\left(M_{12}+\rho_{L} M_{22}\right),
$$




$$
\rho_{R}=\left(M_{21}^{\dagger}-M_{11}^{\dagger} \rho_{L}\right)\left(M_{12}^{\dagger} \rho_{L}-M_{22}^{\dagger}\right)^{-1} .
$$

By eliminating $\rho_{R}$ from (A.7) and (A.8), we obtain

$$
\left(M_{12}+\rho_{L} M_{22}\right)\left(M_{12}^{\dagger} \rho_{L}-M_{22}^{\dagger}\right)=\left(M_{11}+\rho_{L} M_{21}\right)\left(M_{21}^{\dagger}-M_{11}^{\dagger} \rho_{L}\right)
$$

or

$$
\begin{gathered}
M_{11} M_{21}^{\dagger}+M_{12} M_{22}^{\dagger}-\left(M_{11} M_{11}^{\dagger}+M_{12} M_{12}^{\dagger}\right) \rho_{L} \\
+\rho_{L}\left(M_{21} M_{21}^{\dagger}+M_{22} M_{22}^{\dagger}\right)-\rho_{L}\left(M_{21} M_{11}^{\dagger}+M_{22} M_{12}^{\dagger}\right) \rho_{L}=0
\end{gathered}
$$

Similarly, we obtain the relation

$$
\begin{gathered}
M_{11}^{\dagger} M_{12}+M_{21}^{\dagger} M_{22}-\left(M_{11}^{\dagger} M_{11}+M_{21}^{\dagger} M_{21}\right) \rho_{R} \\
+\rho_{R}\left(M_{12}^{\dagger} M_{12}+M_{22}^{\dagger} M_{22}\right)-\rho_{R}\left(M_{12}^{\dagger} M_{11}+M_{22}^{\dagger} M_{21}\right) \rho_{R}=0 .
\end{gathered}
$$

Eliminating $M_{22}$ from (A.5) and (A.6), we obtain

$$
\begin{gathered}
\rho_{L} M_{22} \rho_{R}^{\dagger}=\left(M_{11} \rho_{R}+\rho_{L} M_{21} \rho_{R}-M_{12}\right) \rho_{R}^{\dagger} \\
=\rho_{L}\left(\rho_{L}^{\dagger} M_{11}+\rho_{L}^{\dagger} M_{12} \rho_{R}^{\dagger}-M_{21}\right),
\end{gathered}
$$

so that

$$
\left(1+\rho_{L} \rho_{L}^{\dagger}\right)\left(M_{11}+M_{12} \rho_{R}^{\dagger}\right)=\left(M_{11}+\rho_{L} M_{21}\right)\left(1+\rho_{R} \rho_{R}^{\dagger}\right) .
$$

Similarly, eliminating $M_{11}$ from (A.5) and (A.6), we obtain

$$
\begin{gathered}
\rho_{L}^{\dagger} M_{11} \rho_{R}=\rho_{L}^{\dagger}\left(M_{12}+\rho_{L} M_{22}-\rho_{L} M_{21} \rho_{R}\right) \\
=\left(M_{21}+M_{22} \rho_{R}^{\dagger}-\rho_{L}^{\dagger} M_{12} \rho_{R}^{\dagger}\right) \rho_{R},
\end{gathered}
$$

so that

$$
\left(1+\rho_{L}^{\dagger} \rho_{L}\right)\left(M_{22}-M_{21} \rho_{R}\right)=\left(M_{22}-\rho_{L}^{\dagger} M_{12}\right)\left(1+\rho_{R}^{\dagger} \rho_{R}\right) .
$$

By using the relations (A.13) and (A.15), we obtain

$$
\begin{gathered}
M_{11}^{\prime}=\left(1+\rho_{L} \rho_{L}^{\dagger}\right)^{-1 / 2}\left(M_{11}+M_{12} \rho_{R}^{\dagger}+\rho_{L} M_{21}+\rho_{L} M_{22} \rho_{R}^{\dagger}\right)\left(1+\rho_{R} \rho_{R}^{\dagger}\right)^{-1 / 2} \\
=\left(1+\rho_{L} \rho_{L}^{\dagger}\right)^{-1 / 2}\left(M_{11}+\rho_{L} M_{21}\right)\left(1+\rho_{R} \rho_{R}^{\dagger}\right)^{+1 / 2} \\
=\left(1+\rho_{L} \rho_{L}^{\dagger}\right)^{+1 / 2}\left(M_{11}+M_{12} \rho_{R}^{\dagger}\right)\left(1+\rho_{R} \rho_{R}^{\dagger}\right)^{-1 / 2} \\
M_{22}^{\prime}=\left(1+\rho_{L}^{\dagger} \rho_{L}\right)^{-1 / 2}\left(\rho_{L}^{\dagger} M_{11} \rho_{R}-\rho_{L}^{\dagger} M_{12}-M_{21} \rho_{R}+M_{22}\right)\left(1+\rho_{R}^{\dagger} \rho_{R}\right)^{-1 / 2} \\
=\left(1+\rho_{L}^{\dagger} \rho_{L}\right)^{+1 / 2}\left(M_{22}-M_{21} \rho_{R}\right)\left(1+\rho_{R}^{\dagger} \rho_{R}\right)^{-1 / 2}
\end{gathered}
$$




$$
=\left(1+\rho_{L}^{\dagger} \rho_{L}\right)^{-1 / 2}\left(M_{22}-\rho_{L}^{\dagger} M_{12}\right)\left(1+\rho_{R}^{\dagger} \rho_{R}\right)^{+1 / 2} .
$$

The matrices $\rho_{L}$ and $\rho_{R}$ are obtained as solutions of the equations (A.10) and (A.11), respectively. When the $2 n \times 2 n$ mass matrix $M$ (A.1) is Hermitian, we can set $\rho_{L}=\rho_{R} \equiv \rho$, so that the calculation becomes easier.

When $M$ is not Hermitian, instead of the $n \times n$ mass matrices $M_{11}^{\prime}$ and $M_{22}^{\prime}$, the diagonalization is done for the following Hermitian matrices

$$
\begin{aligned}
& H_{1} \equiv M_{11}^{\prime} M_{11}^{\prime \dagger}=\left(1+\rho_{L} \rho_{L}^{\dagger}\right)^{-1 / 2} \widetilde{H}_{1}\left(1+\rho_{L} \rho_{L}^{\dagger}\right)^{+1 / 2} \\
& H_{2} \equiv M_{22}^{\prime} M_{22}^{\prime \dagger}=\left(1+\rho_{L}^{\dagger} \rho_{L}\right)^{+1 / 2} \widetilde{H}_{2}\left(1+\rho_{L}^{\dagger} \rho_{L}\right)^{-1 / 2}
\end{aligned}
$$

where

$$
\begin{aligned}
& \widetilde{H}_{1}=\left(M_{11}+\rho_{L} M_{21}\right)\left(M_{11}^{\dagger}+\rho_{R} M_{12}^{\dagger}\right), \\
& \widetilde{H}_{2}=\left(M_{22}-M_{21} \rho_{R}\right)\left(M_{22}^{\dagger}-M_{12}^{\dagger} \rho_{L}\right) .
\end{aligned}
$$

We are interested in the diagonalization of (A.22). By using (A.5), we can rewrite (A.22) into

$$
\widetilde{H}_{1}=A+\rho_{L} B
$$

where

$$
\begin{aligned}
& A=M_{11} M_{11}^{\dagger}+M_{12} M_{12}^{\dagger} \\
& B=M_{21} M_{11}^{\dagger}+M_{22} M_{12}^{\dagger}
\end{aligned}
$$

By eliminating $\rho_{L}$ from (A.10) and (A.24), we find that the matrix $\widetilde{H}_{1}$ satisfies the following equations

$$
\widetilde{H}_{1}^{2}-\widetilde{H}_{1}\left(A+B^{-1} D B\right)+A B^{-1} D B-C B=0
$$

where

$$
\begin{aligned}
& C=M_{11} M_{21}^{\dagger}+M_{12} M_{22}^{\dagger} \\
& D=M_{21} M_{21}^{\dagger}+M_{22} M_{22}^{\dagger}
\end{aligned}
$$




\section{References and Footnotes}

[ 1 ] The seesaw mechanism has originally proposed for the purpose of explaining why neutrino masses are so invisibly small: T. Yanagida, in Proc. Workshop of the Unified Theory and Baryon Number in the Universe, edited by A. Sawada and A. Sugamoto (KEK, 1979); M. Gell-Mann, P. Rammond and R. Slansky, in Supergravity, edited by P. van Nieuwenhuizen and D. Z. Freedman (NorthHolland, 1979); R. Mohapatra and G. Senjanovic, Phys. Rev. Lett. 44 (1980) 912.

[ 2 ] For applications of the seesaw mechanism to the quark mass matrix, see, for example, Z. G. Berezhiani, Phys. Lett. 129B (1983) 99; Phys. Lett. 150B (1985) 177; D. Chang and R. N. Mohapatra, Phys. Rev. Lett. 58 (1987) 1600; A. Davidson and K. C. Wali, Phys. Rev. Lett. 59 (1987) 393; S. Rajpoot, Mod. Phys. Lett. A2 (1987) 307; Phys. Lett. 191B (1987) 122; Phys. Rev. D36 (1987) 1479; K. B. Babu and R. N. Mohapatra, Phys. Rev. Lett. 62 (1989) 1079; Phys. Rev. D41 (1990) 1286; S. Ranfone, Phys. Rev. D42 (1990) 3819; A. Davidson, S. Ranfone and K. C. Wali, Phys. Rev. D41 (1990) 208; I. Sogami and T. Shinohara, Prog. Theor. Phys. 66 (1991) 1031; Phys. Rev. D47 (1993) 2905; Z. G. Berezhiani and R. Rattazzi, Phys. Lett. B279 (1992) 124; P. Cho, Phys. Rev. D48 (1994) 5331; A. Davidson, L. Michel, M. L, Sage and K. C. Wali, Phys. Rev. D49 (1994) 1378; W. A. Ponce, A. Zepeda and R. G. Lozano, Phys. Rev. D49 (1994) 4954.

[ 3 ] Y. Koide, Phys. Rev. D49 (1994) 2638.

[ 4 ] H. Harari, H. Haut and J. Weyers, Phys. Lett. B78 (1978) 459; T. Goldman, in Gauge Theories, Massive Neutrinos and Proton Decays, edited by A. Perlumutter (Plenum Press, New York, 1981), p.111; T. Goldman and G. J. Stephenson, Jr., Phys. Rev. D24 (1981) 236; Y. Koide, Phys. Rev. Lett. 47 (1981) 1241; Phys. Rev. D28 (1983) 252; 39 (1989) 1391; C. Jarlskog, in Proceedings of the International Symposium on Production and Decays of Heavy Hadrons, Heidelberg, Germany, 1986 edited by K. R. Schubert and R. Waldi (DESY, Hamburg), 1986, p.331; P. Kaus, S. Meshkov, Mod. Phys. Lett. A3 (1988) 1251; Phys. Rev. D42 (1990) 1863; L. Lavoura, Phys. Lett. B228 
(1989) 245; M. Tanimoto, Phys. Rev. D41 (1990) 1586; H. Fritzsch and J. Plankl, Phys. Lett. B237 (1990) 451; Y. Nambu, in Proceedings of the International Workshop on Electroweak Symmetry Breaking, Hiroshima, Japan, (World Scientific, Singapore, 1992), p.1.

[ 5 ] Y. Koide, Mod. Phys. Lett. A8 (1993) 2071.

[ 6 ] H. Fusaoka and Y. Koide, Mod. Phys. Lett. A10 (1995) 289.

[ 7 ] M. Kobayashi and T. Maskawa, Prog. Theor. Phys. 49 (1973) 652.

[ 8 ] CDF collaboration, F. Abe et al., Phys. Rev. Lett. 73 (1994) 225; Phys. Rev. D50 (1994) 2966.

[ 9 ] A seesaw neutrino mass matrix with a democratic-type matrix $M_{F}$ was first discussed by Goldman and Stephenson in ref. [4]. Their interest, however, was not in the quark mass matrix, and their matrix $M_{F}$ does not include the unit matrix part, which is different from (2.2).

[ 10 ] H. Harari, H. Haut and J. Weyers, Phys. Lett. B78 (1978) 459.

[ 11 ] P. Kaus, S. Meshkov, Mod. Phys. Lett. A3 (1988) 1251; Phys. Rev. D42 (1990) 1863; Y. Nambu, in Proceedings of the International Workshop on Electroweak Symmetry Breaking, Hiroshima, Japan, (World Scientific, Singapore, 1992), p.1.

[ 12 ] Y. Koide, Phys. Rev. Lett. 47 (1981) 1241.

[ 13 ] Particle data group, Phys. Rev. D50 (1994) 1173.

[ 14 ] For the light quark masses $m_{q}(\mu)$, we have used the values at $\mu=1 \mathrm{GeV}$, $m_{u}=5.6 \pm 1.1 \mathrm{MeV}, m_{d}=9.9 \pm 1.1 \mathrm{MeV}$ and $m_{s}=199 \pm 33 \mathrm{MeV}:$ C. A.Dominquez and E. de Rafael, Annals of Physics 174 (1987) 372. However, the absolute values of light quark masses should not be taken solidly because they depend on models. For $m_{c}(\mu)$ and $m_{b}(\mu)$, we have used the value $m_{c}\left(m_{c}\right)=1.26 \pm 0.02 \mathrm{GeV}$ by S. Narison, Phys. Lett. B216 (1989) 191 , and the value $m_{b}\left(m_{b}\right)=4.72 \pm 0.05 \mathrm{GeV}$ by C. A. Dominquez and N. Paver, Phys. Lett. B293 (1992) 197. For the top quark mass, we have used $M_{t}^{\text {pole }}=174 \pm 10_{-12}^{+13} \mathrm{GeV}$ from the CDF experiment [8]. 
[ 15 ] P. Langacker and S. U. Sankar, Phys. Rev. D40 (1989) 1569.

[ 16 ] C. Jarlskog, Phys. Rev. Lett. 55 (1985) 1839; O. W. Greenberg, Phys. Rev. D32 (1985) 1841; I. Dunietz, O. W. Greenberg, and D.-d. Wu, Phys. Rev. Lett. 55 (1985) 2935; C. Hamzaoui and A. Barroso, Phys. Lett. 154B (1985) 202; D.-d. Wu, Phys. Rev. D33 (1986) 860.

[ 17 ] S. L. Glashow, J. Iliopulos and L. Maiani, Phys. Rev. D2 (1970) 1285. 


\section{Figure Captions}

Fig. 1. Masses $m_{i}^{f}(i=1,2,3)$ versus $b_{f}$ for the case of $k=10$ and $K / k=$ 50. The solid and broken lines denote for the cases of $\beta_{f}=0$ and $\beta_{f}=-20^{\circ}$, respectively. The parameters $k$ and $K$ are defined by (2.1). The figure should be taken as that for the quark mass ratios. For the absolute value of quark masses, see a comment on (4.5) in the text.

Fig. 2. Mass ratio $m_{t} / m_{c}$ versus $K / k$ for $k=10$. The curves (a) - (d) denote the cases (a) $\Delta b_{u}=0$, (b) $\Delta b_{u}=+1.00 \times 10^{-3}$ and $\Delta b_{u}=-0.980 \times 10^{-3}$, (c) $\Delta b_{u}=+3.88 \times 10^{-3}$ and $\Delta b_{u}=-3.62 \times 10^{-3}$, (d) $\Delta b_{u}=+10.0 \times 10^{-3}$ and $\Delta b_{u}=-8.53 \times 10^{-3}$. The horizontal lines denote the experimental values $\left(m_{t} / m_{c}\right)_{\text {exp }}=271 \pm 46$.

Fig. 3. Top quark mass $m_{t}$ in unit of $m_{0}$ versus $K / k$ for $k=10$. The curves (a) - (d) denote the cases (a) $\Delta b_{u}=0$, (b) $\Delta b_{u}=+1.00 \times 10^{-3}$ and $\Delta b_{u}=-0.980 \times 10^{-3},\left(\mathrm{c}_{+}\right) \Delta b_{u}=+3.88 \times 10^{-3},\left(\mathrm{c}_{-}\right) \Delta b_{u}=-3.62 \times 10^{-3},\left(\mathrm{~d}_{+}\right)$ $\Delta b_{u}=+10.0 \times 10^{-3}$, and $\left(\mathrm{d}_{-}\right) \Delta b_{u}=-8.53 \times 10^{-3}$.

Fig. 4. Mass ratios $m_{s} / m_{d}$ and $m_{b} / m_{s}$ versus $\beta_{d}$ for $b_{d}=-0.90$ (a dotted line), $b_{d}=-1.0$ (a solid line) and $b_{d}=-1.1$ (a broken line) in the case of $k=10$ and $K / k=50$.

Fig. 5. Kobayashi-Maskawa matrix elements $\left|V_{u s}\right|,\left|V_{c b}\right|$ and $\left|V_{u b}\right|$ versus $\beta_{d}$ in the case of $k=10, K / k=50, b_{u}=-1 / 3$ and $\beta_{u}=0$. 
This figure "fig1-1.png" is available in "png" format from: http://arxiv.org/ps/hep-ph/9505201v3 
This figure "fig2-1.png" is available in "png" format from: http://arxiv.org/ps/hep-ph/9505201v3 
This figure "fig1-2.png" is available in "png" format from: http://arxiv.org/ps/hep-ph/9505201v3 
This figure "fig2-2.png" is available in "png" format from: http://arxiv.org/ps/hep-ph/9505201v3 
This figure "fig1-3.png" is available in "png" format from: http://arxiv.org/ps/hep-ph/9505201v3 